\title{
Classification Using Neural Networks and Deep Learning
}

Title: Classification Using Neural Networks and Deep Learning

Student Name: Raju (rsingh80@asu.edu)

\begin{abstract}
:
This report is an insight into the world of deep learning and CNN networks. It is an attempt to perform classification using neural network and deep learning for a given dataset (which is a subset from the MNIST dataset). The MNIST dataset contains 70,000 images of handwritten digits, divided into 60,000 training images and 10,000 testing images.
\end{abstract}

Parameter for CNN:

- The input size is the size of the image (28x28).

- The first hidden layer is a convolutional layer, with 6 feature maps. The convolution kernels are of $3 \times 3$ in size. Use stride 1 for convolution.

- The convolutional layer is followed by a max pooling layer. The pooling is $2 \times 2$ with stride 1.

- After max pooling, the layer is connected to the next convolutional layer, with 16 feature maps. The convolution kernels are of $3 \times 3$ in size. Use stride 1 for convolution.

- The second convolutional layer is followed by a max pooling layer. The pooling is $2 \times 2$ with stride 1.

- After max pooling, the layer is fully connected to the next hidden layer with 120 nodes and relu as the activation function.

- The fully connected layer is followed by another fully connected layer with 84 nodes and relu as the activation function, then connected to a softmax layer with 10 output nodes (corresponding to the 10 classes).

Tasks:

1. Run the baseline code (as provided) and report the accuracy.

2. Change the kernel size to $5 * 5$, redo the experiment, plot the learning errors along with the epoch, and report the testing error and accuracy on the test set.

3. Change the number of the feature maps in the first and second convolutional layers, redo the experiment, plot the learning errors along with the epoch, and report the testing error and accuracy on the test set. 
Algorithm:

Convolution Neural Network

Resources:

Google Colab [https://colab.research.google.com ]

MNIST dataset

Language:

Python 3.6.8

\section{Keywords:}

Python, mnist dataset, CNN, neural network, pandas, numpy, array, softmax, relu, strides, hidden layers, input output layer.

[body]

\section{Introduction:}

Plot the training error and the testing error as a function of the learning epochs. Change some of the hyper-parameters (the kernel size, the number of feature maps, etc), and then repeat the experiment and plot training and testing errors under the new setting.

Convolution Neural Network $(\mathrm{CNN})$ is a machine learning technique, used for image classification. In this example, $\mathrm{CNN}$ is used with keras with tensorflow backend for the MNIST digit recognition tasks.

Convolution expresses as:

$$
\begin{aligned}
& g(x, y)=\omega * f(x, y)=\sum_{s=-a}^{a} \sum_{t=-b}^{b} \omega(s, t) f(x-s, y-t) \text {, } \\
& \text { where } g(x, y) \text { is the filtered image, } f(x, y) \text { is the original image, wis the filter kernel. Every element of the filter kernel is considered by }-a \leq s \leq a \text { and }-b \leq t \leq b \text {. }
\end{aligned}
$$

Scope 1: The following steps are executed in sequence:

1. Prepare the data for the baseline code.

2. Building and compiling the model.

3. Training and evaluating the model.

4. Report the accuracy.

In the baseline code, import the libraries used in the CNN. It uses keras and tensorflow as a backend. Set the batch size, number of classes, and epochs. The image has a dimension of 28, 28 (rows, columns). Next, load the data, create the channel and convert the class vectors to binary class matrices. Run the evaluation model with kernel size as $3 * 3$, activation as 'ReLU'. Further, Further to building, compile the model, report the accuracy/error, plot the graph. 


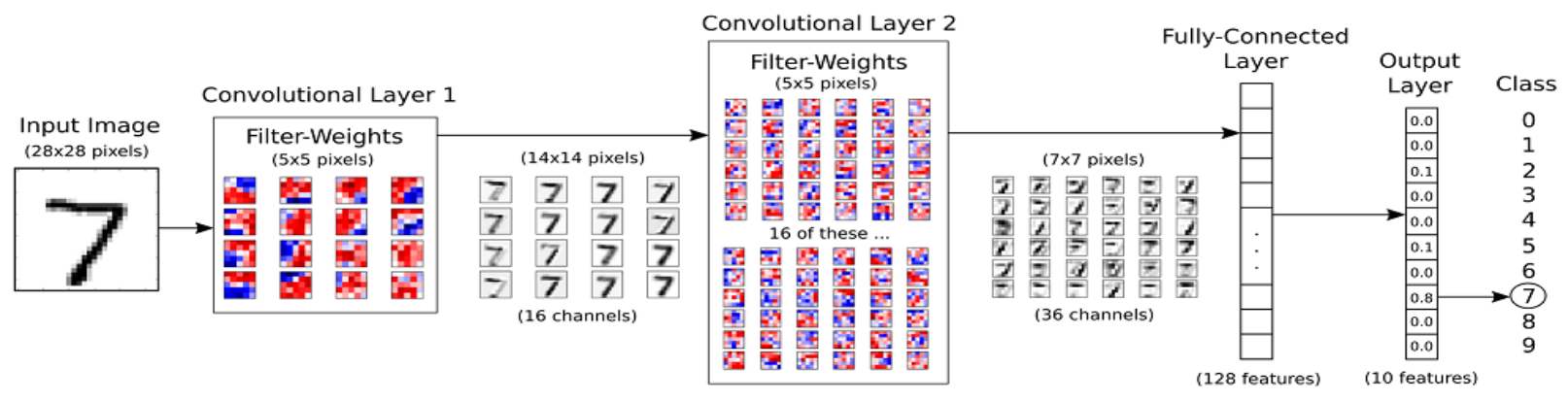

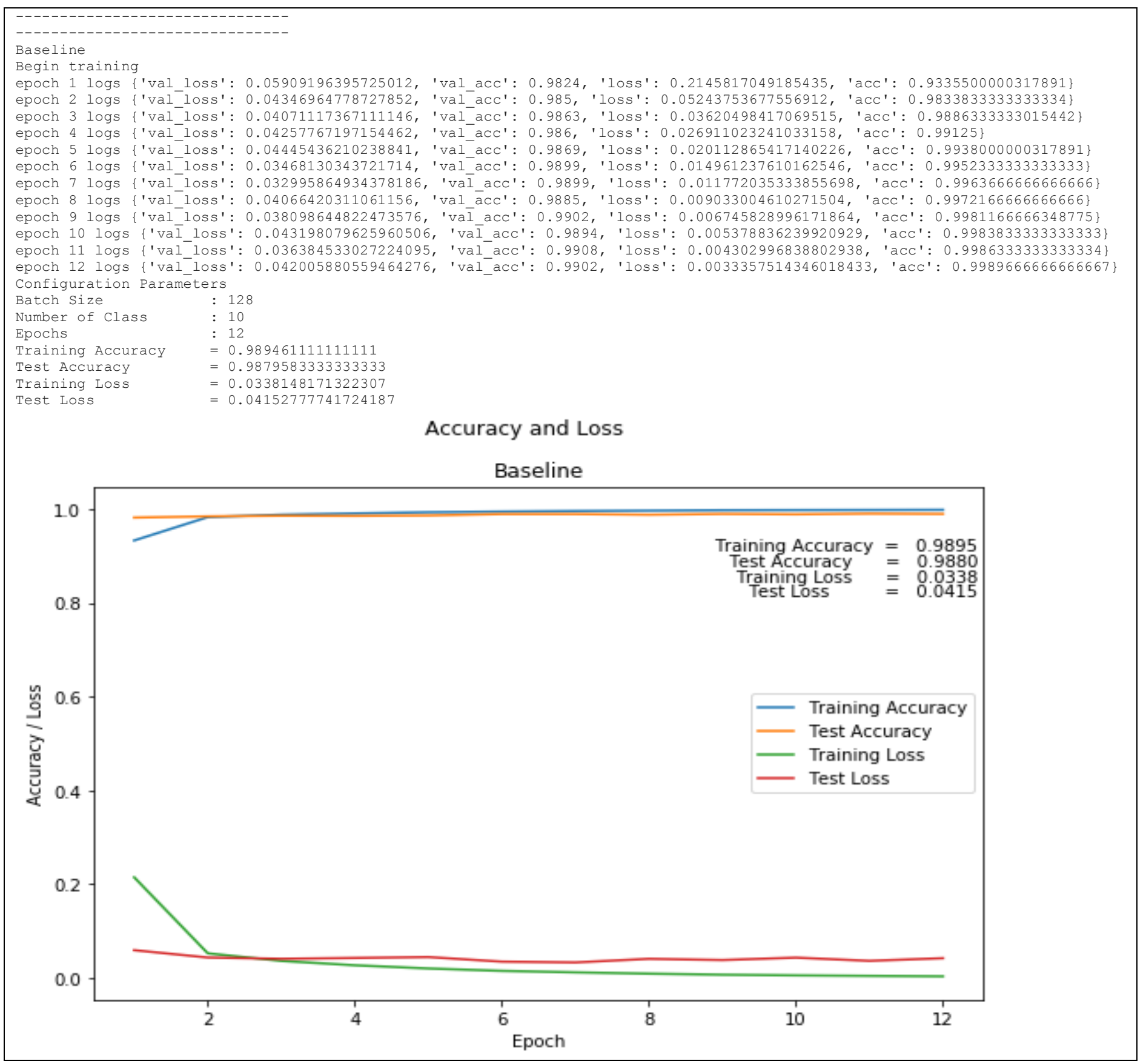


Scope 2: In this task, following steps are executed:

1. Change the kernel size to $5 * 5$

2. Redo the experiment.

3. Plot the learning errors along with the epoch

4. Report the testing error and accuracy on the test set.

Update the code, to meet the kernel size as:

modelE1 .add(Conv2D $(6$, kernel_size $=(5,5)$,activation='relu',input_shape=input_shape,strides $=(1,1)))$

In this task, the kernel size is changed to $5^{*} 5$ and redo the experiment. The kernel size is increased to learn large spatial filters, and reduce the volume size.
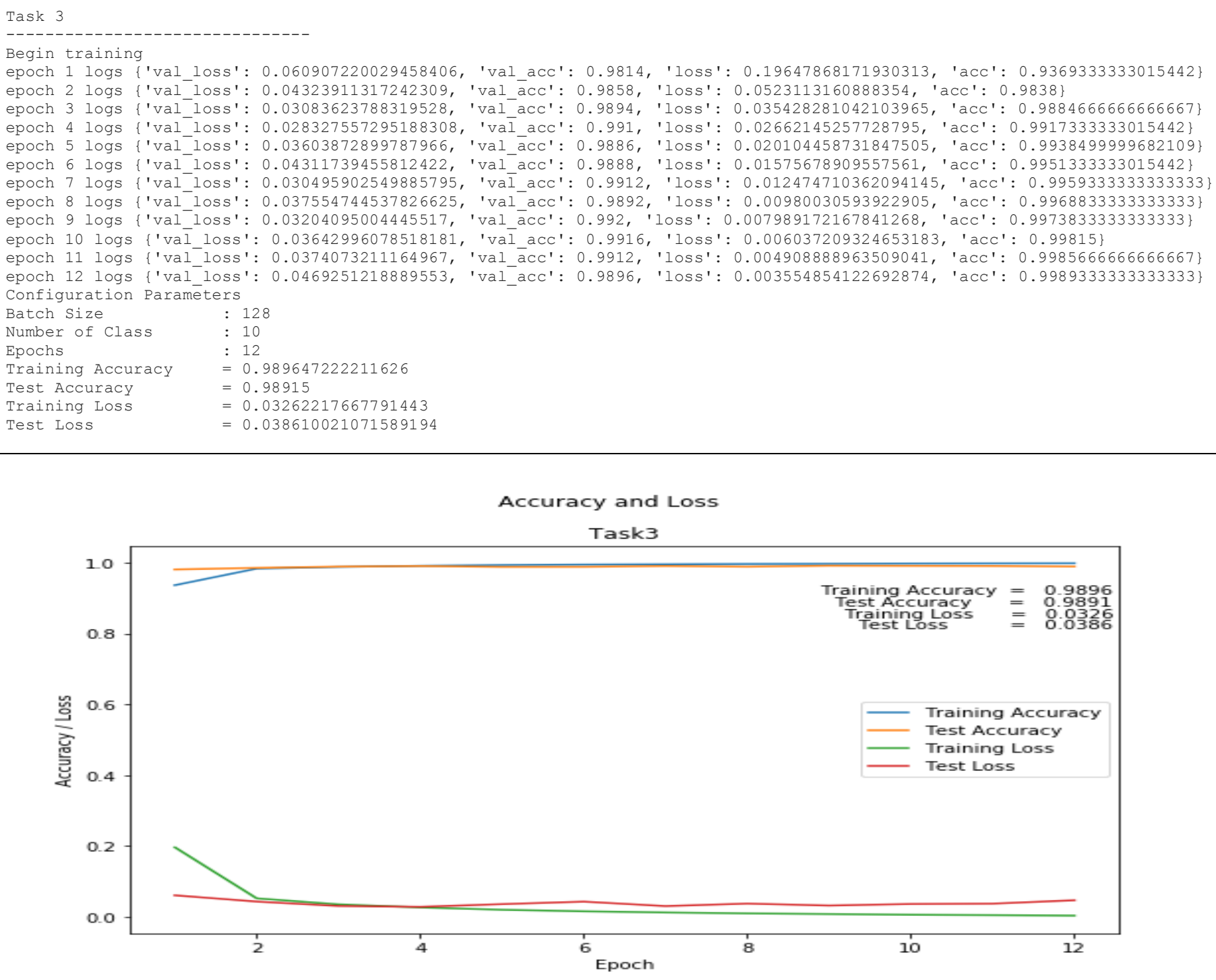
Scope 3: In this, following steps are executed.

1. Change the number of the feature maps in the first and second convolutional layer

2. Redo the experiment

3. Plot the learning errors along with the epoch,

4. Report the testing error and accuracy on the test set.

In this task, change the number of the feature maps in the first and second convolutional layers with values 32 and 64, with kernel(5,5). Plot the learning errors along with the epoch, and report the testing error and accuracy on the test set. Convolution is applied on the input data using convolution filter to produce a feature map. Aggregate the convolution results in the feature map.
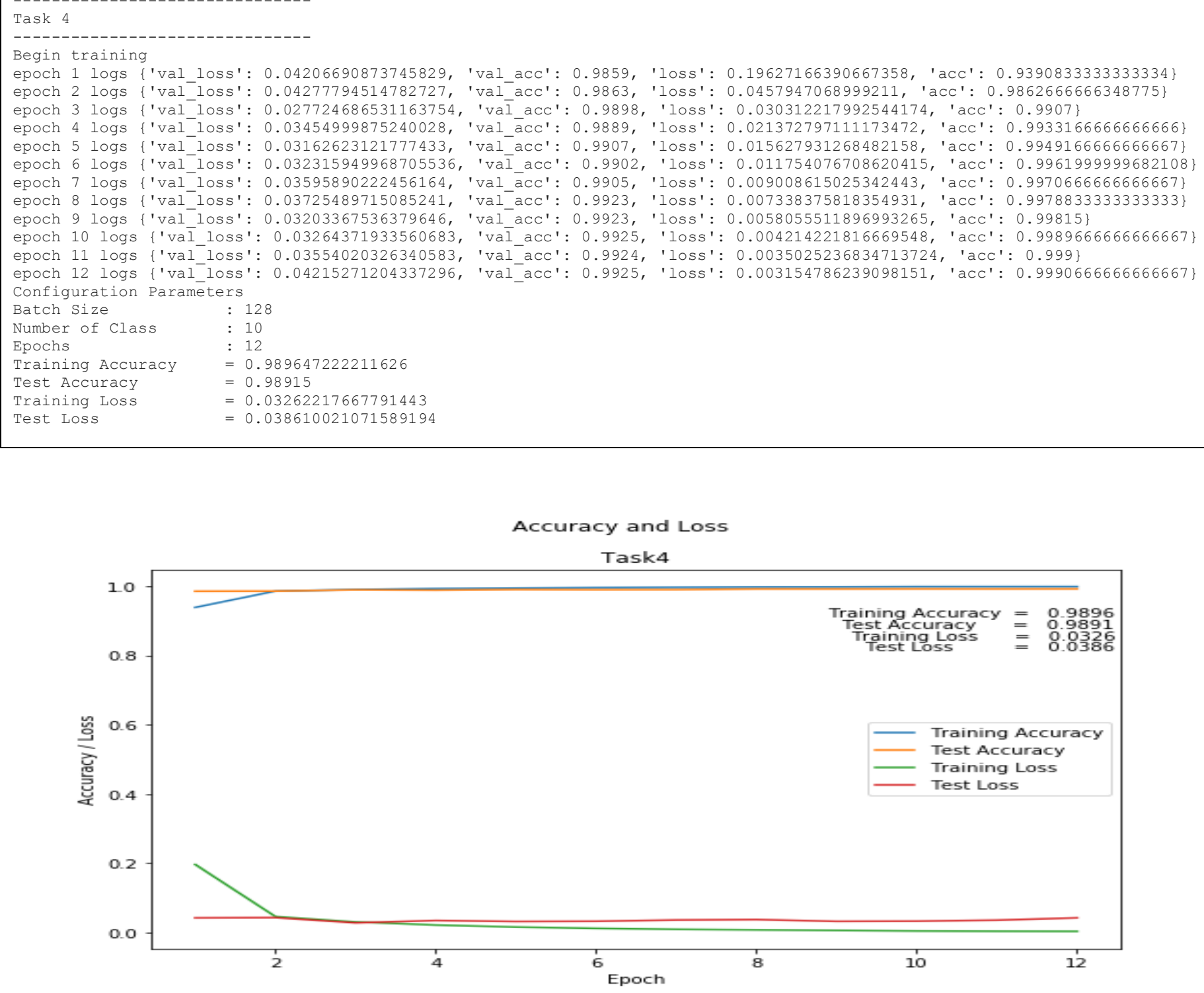


\section{Reference:}

[1] K. I. Kim and K. M. Lee, "Context-aware information provisioning for vessel traffic service using rule-based and deep learning techniques," International Journal of Fuzzy Logic and Intelligent Systems, vol. 18, no. 1, pp. 13-19, 2018. http://doi.org/10.5391/IJFIS.2018.18.1.13

[2] H. W. Lee, N. R. Kim, and J. H. Lee, "Deep neural network self-training based on unsupervised learning and dropout," International Journal of Fuzzy Logic and Intelligent Systems, vol. 17, no. 1, pp. 1-9, 2017. https://doi.org/10.5391/IJFIS.2017.17.1.1

[3] W. A. Bergerud, "Introduction to regression models: with worked forestry examples," British Columbia Ministry of Forests, Victoria, Canada, 1996.

[4] S. Sabour, N. Frosst, and G. E. Hinton, "Dynamic routing between capsules," Advances in Neural Information Processing Systems, vol. 30, pp. 3859-3869, 2017.

[5] A. Alsaafin and A. Elnagar, "A minimal subset of features using feature selection for handwritten digit recognition," Journal of Intelligent Learning Systems and Applications, vol. 9, no. 4, pp. 55-68, 2017. https://doi.org/10.4236/jilsa. 2017.94006

[6] J. Engel, "Polytomous logistic regression," Statistica Neerlandica, vol. 42, no. 4, pp. 233-252, 1988. https: //doi.org/10.1111/j.1467-9574.1988.tb01238.x

[7] D. W. Hosmer and S. Lemeshow, Applied Logistic Regression, 2nd ed. Hoboken, NJ: John Wiley \& Sons, 2005. https://doi.org/10.1002/0471722146

[8] C. C. J. Kuo, "Understanding convolutional neural networks with a mathematical model," Journal of Visual Communication and Image Representation, vol. 41, pp. 406- 413, 2016. https://doi.org/10.1016/j.jvcir.2016.11.003

[9] P. Y. Simard, D. Steinkraus, and J. C. Platt, "Best practices for convolutional neural networks applied to visual document analysis," in Proceedings of 7th International Conference on Document Analysis and Recognition, Edinburgh, UK, 2003, pp. 958-962.

[10] A. Krizhevsky, I. Sutskever, and G. Hinton, "ImageNet classification with deep convolutional neural networks," Advances in Neural Information Processing Systems, vol. 25, pp. 1097-1105, 2012.

[11] M. Ranzato, F. J. Huang, Y. L. Boureau, and Y. LeCun, "Unsupervised learning of invariant feature hierarchies with applications to object recognition," in Proceedings of IEEE Conference on Computer Vision and Pattern Recognition, Minneapolis, MN, 2007, pp. 1-8. https://doi.org/10.1109/CVPR.2007.383157

[12] M. D. Zeiler and R. Fergus, "Stochastic pooling for regularization of deep convolutional neural networks," 2013, Available https://arxiv.org/abs/1301.3557 
[13] I. J. Goodfellow, D. Warde-Farley, M. Mirza, A. Courville, and Y. Bengio, "Maxout networks," in Proceedings of the 30th International Conference on International Conference on Machine Learning, Atlanta, GA, 2013, pp. 1319- 1327.

[14] N. Srivastava, G. Hinton, A. Krizhevsky, I. Sutskever, and R. Salakhutdinov, "Dropout: a simple way to prevent neural networks from overfitting," Journal of Machine Learning Research, vol. 15, no. 1, pp. 1929-1958, 2014.

[15] F. Lauer, C. Y. Suen, and G. Bloch, "A trainable feature extractor for handwritten digit recognition," Pattern Recognition, vol. 40, no. 6, pp. 1816-1824, 2007. https://doi.org/10.1016/j.patcog.2006.10.011

[16] C. Y. Lee, S. Xie, P. Gallagher, Z. Zhang, and Z. Tu, "Deeply-supervised nets," in Proceedings of the 18th International Conference on Artificial Intelligence and Statistics, San Diego, CA, 2015, pp. 562-570.

[17] K. He, X. Zhang, S. Ren and J. Sun, "Spatial pyramid pooling in deep convolutional networks for visual recognition," IEEE Transactions on Pattern Analysis and Machine Intelligence, vol. 37, no. 9, pp. 1904-1916. https://doi.org/10.1109/TPAMI.2015.2389824

[18] R. Girshick, "Fast R-CNN," in Proceedings of 2015 IEEE International Conference on Computer Vision, Santiago, Chile, 2015, pp. 1440-1448.

[19] C. Couprie, C. Farabet, L. Najman, and Y. LeCun, "Indoor semantic segmentation using depth information," 2013, Available https://arxiv.org/abs/1301.3572 